\title{
Matrix Metalloproteinase-7 Regulates Cleavage of Pro-Nerve Growth Factor and Is Neuroprotective following Kainic Acid-Induced Seizures
}

\author{
Audrey P. Le and Wilma J. Friedman \\ Department of Biological Sciences, Center for Molecular and Behavioral Neuroscience, Rutgers University, Newark, New Jersey 07102
}

The neurotrophin nerve growth factor (NGF) regulates neuronal growth, differentiation, and survival during development. However, the precursor of NGF, proNGF, is a potent apoptotic ligand for the $\mathrm{p} 75$ neurotrophin receptor ( $\left.\mathrm{p} 75^{\mathrm{NTR}}\right)$-sortilin complex. The mechanisms that regulate cleavage of proNGF, therefore, are critical determinants of whether this factor promotes neuronal survival or death. In this study, we demonstrate that, following kainic acid-induced seizures, the proNGF processing enzyme matrix metalloproteinase 7 (MMP-7) and its inhibitor TIMP-1 (tissue inhibitor of matrix metalloproteinase 1) are regulated in a manner that prevents proneurotrophin cleavage and leads to increased proNGF in the extracellular milieu. Furthermore, we demonstrate both in vitro and in vivo that exogenous MMP-7 enhances proNGF cleavage and provides neuroprotection following kainic acid treatment. These data demonstrate that increased extracellular proNGF levels following seizures are stabilized by altered MMP-7 enzymatic activity, leading to increased neuronal death via activation of $\mathrm{p} 75^{\mathrm{NTR}}$.

\section{Introduction}

Nerve growth factor (NGF) influences neuronal survival through binding with the TrkA receptor (Levi-Montalcini, 1964, 1987). However, the NGF precursor, proNGF, can function in a biologically opposite manner, inducing apoptosis by binding with the p75 neurotrophin receptor ( $\mathrm{p} 75^{\mathrm{NTR}}$ )-sortilin complex. p75 ${ }^{\mathrm{NTR}}$ mediated apoptosis has been described in various systems, including oligodendrocyte and hippocampal neuron cultures (Casaccia-Bonnefil et al., 1996; Friedman, 2000), as well as in vivo conditions such as seizure (Troy et al., 2002) and corticospinal axotomy (Giehl et al., 2001). Increased proNGF and p $75^{\text {NTR }}$ have been detected in the brains of Alzheimer's patients (Peng et al., 2004), after spinal cord injury (Beattie et al., 2002), and in the hippocampus after seizure (Volosin et al., 2008). Furthermore, disrupting proNGF-p $75^{\text {NTR }}$ interaction after corticospinal axotomy or seizure significantly reduced cell death (Harrington et al., 2004; Volosin et al., 2008). These studies suggest that the apoptotic consequence of $\mathrm{p} 75^{\mathrm{NTR}}$ signaling may be dependent upon the availability of proNGF.

Because proNGF is cleaved to generate NGF, the proteolysis of proNGF may be a critical checkpoint in determining the extent of cell death after injury. What determines whether secreted proNGF is cleaved or stabilized in its pro- form remains unclear. ProNGF can

Received Aug. 10, 2011; revised Oct. 31, 2011; accepted Nov. 2, 2011.

Author contributions: W.J.F. and A.P.L. designed research; A.P.L. performed research; A.P.L. and W.J.F. analyzed data; A.P.L. wrote the paper.

This work was supported by NIH Grant NS045556. We thank Matthew Wilkins for technical assistance, and Soyoung Choi and Marta Volosin for valuable input.

Correspondence should be addressed to Dr. Wilma J. Friedman, Department of Biological Sciences, Rutgers University, 225 University Avenue, Newark, NJ 07102. E-mail: wilmaf@andromeda.rutgers.edu.

DOI:10.1523/JNEUROSCI.4128-11.2012

Copyright $\odot 2012$ the authors $\quad 0270-6474 / 12 / 320703-10 \$ 15.00 / 0$ be extracellularly processed to NGF by the enzyme matrix metalloproteinase 7 (MMP-7) (Smith et al., 1995; Lee et al., 2001). The MMPs constitute a family of enzymes responsible for degrading the extracellular matrix (Birkedal-Hansen, 1993; Wojtowicz-Praga et al., 1997). MMP-7 in particular functions in tissue remodeling and wound healing (Parks and Shapiro, 2001; Buhler et al., 2009), and its activity has been shown to be necessary for proNGF to NGF conversion in vivo (Kendall et al., 2009). Recently, MMP-7 production and activity were found to be significantly decreased in rat and clinical patient models of diabetic retinopathy, leading to proNGF accumulation and retinal neurodegeneration via p $75^{\text {NTR }}$ (Ali et al., 2011).

MMP-7 activity is inhibited by tissue inhibitor of metalloproteinase 1 (TIMP-1). TIMP-1 binds to the pro- form of MMP-7, blocking substrate interaction and the cleavage required for activation (Gogly et al., 2009). TIMP-1 functions in many CNS processes, such as maintaining the blood-brain barrier and mediating excitotoxic stress (Tan et al., 2003). Following seizure, however, TIMP-1 mRNA and protein were strongly upregulated in the forebrain and hippocampus, coinciding with areas of maximal cell death (Rivera et al., 1997). Conversely, TIMP-1 knock-out mice demonstrated resistance to seizure-induced cell death (Jourquin et al., 2005). The upregulation of TIMP-1 after seizure and other pathological conditions suggests that an imbalance between MMP-7 and TIMP-1 may influence neuronal death by enhancing levels of proNGF.

We have investigated the expression and activity of MMP-7 and TIMP-1 in the hippocampus following kainic acid-induced injury, the relationship of this enzyme-inhibitor system to proNGF processing, and the consequences for $\mathrm{p} 75^{\mathrm{NTR}}$-mediated apoptosis in vitro and in vivo. We demonstrate that seizures decrease MMP-7 and increase TIMP-1, attenuating cleaving activity and leading to increased proNGF and $\mathrm{p} 75^{\mathrm{NTR}}$ activation. Furthermore, providing exogenous MMP-7 after injury promoted proNGF 
cleavage and neuroprotection, suggesting a novel regulatory mechanism for $\mathrm{p} 75^{\mathrm{NTR}}$ mediated apoptosis.

\section{Materials and Methods}

Hippocampal slice cultures. Organotypic hippocampal cultures were prepared as previously described (Stoppini et al., 1991). Postnatal day 7 (P7) rat pups were killed by exposure to $\mathrm{CO}_{2}$ and soaked in $80 \%$ ethanol for $10 \mathrm{~min}$. Their brains were removed under sterile conditions and placed into HBSS plus glucose (100 ml of HBSS with $0.6 \mathrm{~g}$ of glucose). Hippocampi were removed in HBSS and set on sterile Whatman chromatography paper, and $300 \mu \mathrm{m}$ slices were prepared using a Mcllwain tissue chopper. Slices that retained the cytoarchitecture of the hippocampus were placed onto Millipore inserts suspended in $1.5 \mathrm{ml}$ of serum-containing media (MEM with 25\% horse serum, $6 \mathrm{mg} / \mathrm{ml}$ glucose, 1 $\mu \mathrm{M}$ penicillin-streptomyocin). Cultures were maintained at $37^{\circ} \mathrm{C}$ in $95 \% \mathrm{O}_{2}$ and $5 \% \mathrm{CO}_{2}$ for $7 \mathrm{~d}$. Afterward, the media was changed to serumfree media (SFM) (containing 1:1 F12/MEM with $6 \mathrm{mg} / \mathrm{ml}$ D-glucose, $100 \mu \mathrm{g} / \mathrm{ml}$ transferrin, $25 \mu \mathrm{g} / \mathrm{ml}$ insulin, $20 \mathrm{~nm}$ progesterone, $60 \mu \mathrm{M} \mathrm{pu}-$ trescine, $30 \mathrm{~nm}$ sodium selenide) for $24 \mathrm{~h}$. Slices were treated with $(+)$-5-methyl-10,11-dihydro$5 H$-dibenzo $[a, d]$ cyclohepten-5,10-imine maleate (MK-801) (10 $\mu \mathrm{M}$; Tocris Bioscience) for 30 min to block NMDA receptor activity, and then 5 $\mu \mathrm{M}$ kainic acid (KA). After $1 \mathrm{~h}$, KA was washed out and slices were returned to fresh SFM containing $10 \mu \mathrm{M}$ MK-801. For analysis of NGF secretion, media samples also received $1 \mu \mathrm{g} / \mathrm{ml}$ TrkA/Fc chimera (R\&D Systems). Slices were fixed for immunohistochemistry or lysed for Western blot analysis at 2, 4, 8, and $24 \mathrm{~h}$. Control slices were prepared similarly but were treated with vehicle instead of KA. Imaging of immunolabeled slices was performed on a laser-scanning point confocal microscope (LSM-510; CarlZeiss) with an optical slice thickness of $\sim 1 \mu \mathrm{m}$.

Propidium iodide assay. Propidium iodide (PI) nucleic acid stain $(5 \mu \mathrm{g} / \mathrm{ml})$ was added to the slice cultures as an indicator of cell death. The amount of cell death was measured as the difference between PI fluorescence intensity at each time point, subtracted from the intensity of the slice after saturation with KA for $24 \mathrm{~h}$ (max intensity). Fluorescence intensity was measured using Adobe Photoshop CS4 (Adobe Systems). For all samples at all time points, images were taken of the whole hippocampus at equal magnification, exposure times, and camera parameters (Hammamatsu Orca).

Animals and kainic acid-induced seizures. Adult male Sprague Dawley rats (250-275 g) were injected with $\mathrm{KA}(10 \mathrm{mg} / \mathrm{kg}$, i.p. $)$ for induction of status epilepticus (SE). After $1 \mathrm{~h}$ of demonstrating stage 5 SE behavior (Racine, 1972), rats were treated with diazepam (10 mg/ kg, i.p.; Henry Schein) and phenytoin (50 mg/ kg, i.p.; Sigma-Aldrich) to stop seizure activity. Control rats received all the same treatments except they were injected with saline instead of KA. Animals not displaying SE were not included in this study. During recovery, the animals were injected subcutaneously with Hartmann's solu-

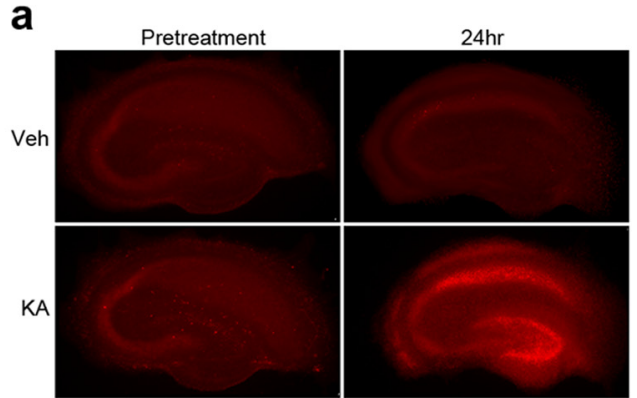

b



C
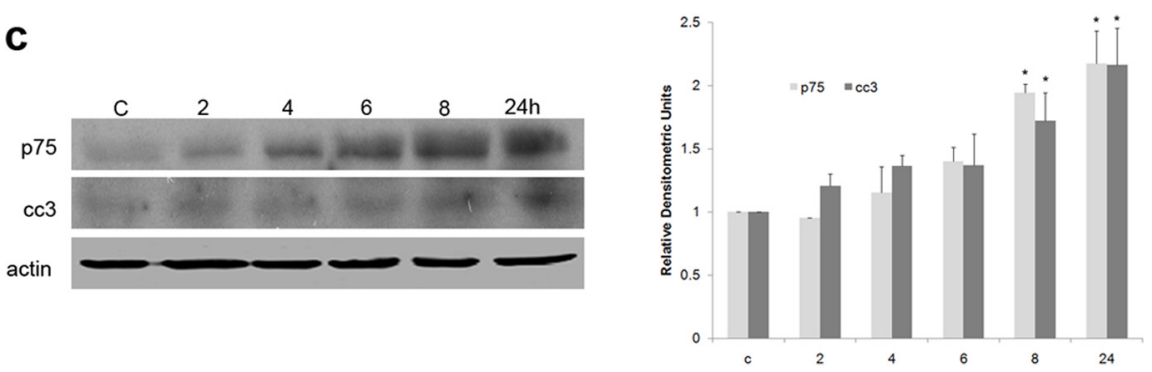

d
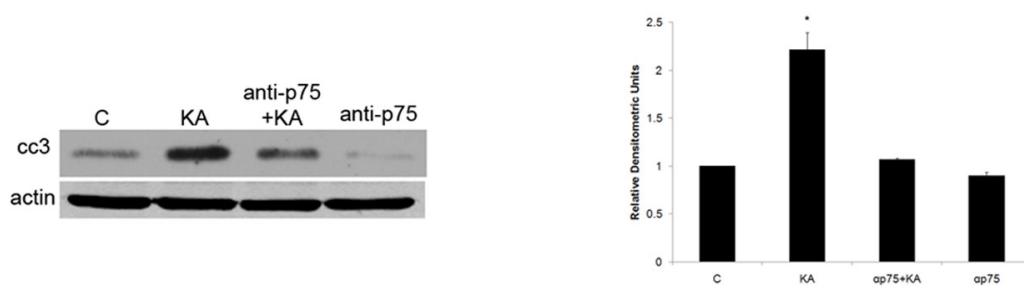

Figure 1. Organotypic hippocampal slice cultures show p $75^{\text {NTR }}$-mediated cell death induced by KA. $\boldsymbol{a}$, Slices from P7 rat pups were cultured for $7 \mathrm{~d}$ with $50 \%$ media changes every $3 \mathrm{~d}$. On day 7 , slices were placed into SFM containing $5 \mu \mathrm{g} / \mathrm{ml} \mathrm{PI}$. After $1 \mathrm{~d}$, pretreatment photographs of each slice were taken (left column). Slices were treated then with $10 \mu \mathrm{l}$ of MK-801 and $1.5 \mu \mathrm{l}$ of vehicle or $5 \mu \mathrm{M} \mathrm{KA}$ (final concentration). After $1 \mathrm{~h}$, media was changed to fresh SFM with MK-801. Photographs were again taken $24 \mathrm{~h}$ following return to SFM. Only slices that received KA treatment demonstrated PI uptake $24 \mathrm{~h}$ after kainic acid treatment, particularly in the CA1 and dentate. $\boldsymbol{b}$, High magnification of CA1 showing $\mathrm{p} 75^{\mathrm{NTR}}$ immunodetection and PI staining after kainic acid treatment. Note the PI-positive nuclei surrounded by $\mathrm{p} 75^{\mathrm{NTR}}$ cytoplasmic staining, indicating dying cells are also positive for $\mathrm{p} 75^{\mathrm{NTR}}$. Photographs show representative staining from five independent experiments. Scale bar, $25 \mu \mathrm{m}$. c, Following KA treatment, slice culture tissue was harvested at 2, 4, 6, 8, and $24 \mathrm{~h}$, lysed in buffer containing protease inhibitors, and subjected to Western blot analysis to examine levels of $p 75^{\text {NTR }}$ and cc 3 compared with untreated control. By $8 \mathrm{~h}$ after KA treatment, levels of both $\mathrm{p} 75^{\mathrm{NTR}}$ and activated caspase had doubled compared with basal levels. Error bars represent SEM. ${ }^{*} p=0.004$ relative to control. Data were analyzed using ANOVA, followed by Student-Newman-Keuls post hoc test. $\boldsymbol{d}$, Cell death following kainic acid treatment is p $75^{\text {NTR }}$ dependent. Slice cultures treated with a blocking antibody to $75^{\text {NTR }}$ at the time of KA treatment show levels of caspase 3 activation comparable with control, indicating p $75^{\text {NTR }}$ is required for KA-induced caspase activation. Error bars represent SEM. ${ }^{*} p=$ 0.002 relative to control. Data were analyzed using ANOVA, followed by Student-Newman-Keuls post hoc test. Each graph represents the densitometric index of immunoblots from three independent experiments normalized to actin and expressed relative to control levels. 
a

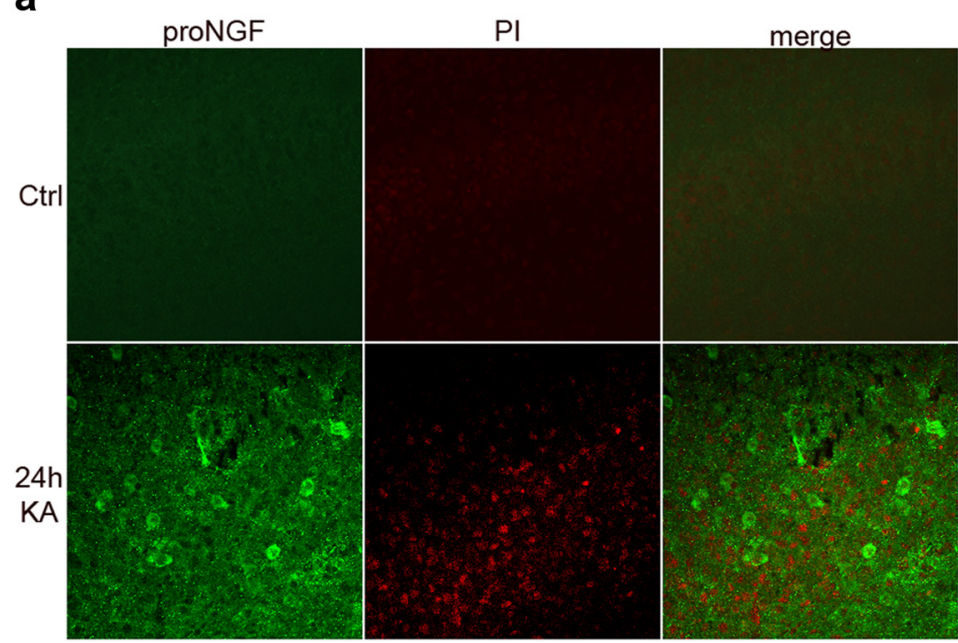

b

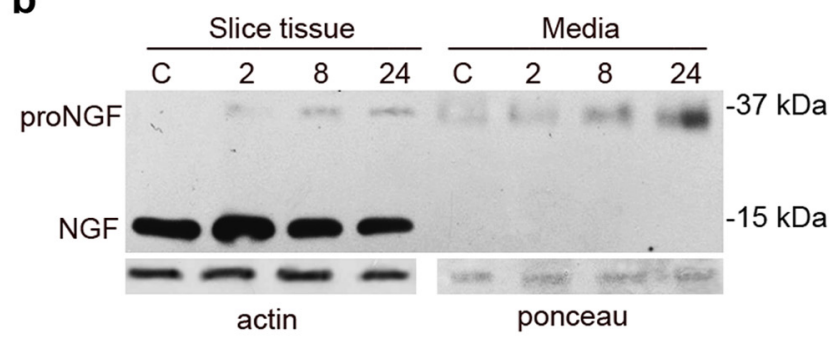

C


Figure 2. proNGF is upregulated and released after kainic acid treatment in slice culture. $\boldsymbol{a}$, proNGF was undetected in CA1 under control conditions (top row). However, in response to KA treatment, proNGF was highly upregulated throughout the CA1, near areas of PI-positive staining. Western analysis $(\boldsymbol{b})$ and quantification $(\boldsymbol{c})$ of tissue from slice cultures detected very low levels of proNGF in control conditions. However, KA induced a 1.5-fold increase in proNGF detection in slice tissue by $8 \mathrm{~h}$ following treatment. Error bars represent SEM. ${ }^{*} p=0.031$ relative to control. Mature NGF was strongly detected in both control and treated conditions but did not show significant differences in expression levels in response to KA. In culture medium, low levels of proNGF were found in control conditions but were nearly doubled by $8 \mathrm{~h}$ following KA stimulation, reaching 2.5 -fold increase by $24 \mathrm{~h}$. Extracellular mature NGF was not detected. Error bars represent SEM. ${ }^{*} p=0.047$ relative to control. Data were analyzed using ANOVA, followed by Student-Newman-Keuls post hoc test. Each graph represents the densitometric index of immunoblots from three independent experiments normalized to actin and expressed relative to control levels.

tion (130 mm NaCl, 4 mm KCl, 3 mm CaCl, 28 mm lactate; $1 \mathrm{ml} / 100$ g) twice daily until they were capable of eating and drinking ad libitum.

For infusion experiments, rats were cannulated 1 week before the induction of seizures. Rats were anesthetized with ketamine hydrochloride (50 $\mathrm{mg} / \mathrm{kg}$ ) and xylazine $(10 \mathrm{mg} / \mathrm{kg})$ and placed in a stereotaxic apparatus for cannula implantation into the dorsal hippocampus with the following coordinates: bregma, - $3.1 \mathrm{~mm}$; lateral, $2.0 \mathrm{~mm}$; and depth, $3.1 \mathrm{~mm}$ (Paxinos and Watson, 1986). Cannulas were fixed to the skull with a screw and dental cement. MMP-7 ( $1 \mu \mathrm{g})$ was infused on one side of the brain and saline on the other side at a rate of $0.5 \mu \mathrm{l} / \mathrm{min}$ immediately following the seizure, and twice daily thereafter until the rats were killed. Animals found to have an incorrectly placed cannula upon histological examination were excluded.

All animal studies were conducted using the National Institutes of Health guidelines for the ethical treatment of animals with approval of the Rutgers Animal Care and Facilities Committee.
Analysis of CSF. At 1 and $3 \mathrm{~d}$ after KA-induced seizure, animals were anesthetized with ketamine/xylazine. CSF (40-100 $\mu$ l per animal) was collected from the cerebello-medullar cisterna using a 25 gauge needle into tubes containing protease inhibitors, snap frozen, and stored at $-80^{\circ} \mathrm{C}$ until analysis. Only CSF samples that did not contain blood contamination were used for Western blot analysis.

Immunohistochemistry. Animals were anesthetized with ketamine/xylazine and perfused transcardially with saline followed by $4 \%$ paraformaldehyde. The brains were removed and postfixed in $4 \%$ paraformaldehyde for $2 \mathrm{~h}$, and cryoprotected in $30 \%$ sucrose overnight. Sections $(12 \mu \mathrm{m})$ were cut on a cryostat (Leica) and mounted onto charged slides. Sections were blocked in $\mathrm{PBS} / 10 \%$ goat serum and permeabilized with PBS/0.3\% Triton X-100, and then exposed to primary antibodies overnight at $4^{\circ} \mathrm{C}$ in $\mathrm{PBS} / 0.3 \%$ Triton $\mathrm{X}-100$. Slides were then washed three times in PBS, exposed for $1 \mathrm{~h}$ at room temperature to secondary antibodies coupled to different fluorophores, and washed again in PBS in the presence of DAPI $\left(4^{\prime}, 6^{\prime}\right.$ diamidino-2-phenylindole) (Sigma-Aldrich; 1:500 dilution). Sections were coverslipped with antifading medium (ProLong Gold; Invitrogen) and analyzed by fluorescence microscopy (Nikon). Primary antisera were as follows: anticleaved caspase 3 (cc3) (1:500; Cell Signaling Technology), anti-NGF (Sigma-Aldrich; 1:1000 dilution), anti-proNGF (generously provided by Barbara Hempstead, Weill Cornell Medical College, New York, NY), anti-MMP-7 (GeneTex; 1:1000 dilution), anti-TIMP-1 (GeneTex; 1:500 dilution), and anti-p75 (9651; 1:500 dilution; generously provided by Moses Chao, Skirball Institute, New York University, New York, NY) or 192 IgG (Millipore Bioscience Research Reagents; 1:1000 dilution). No immunostaining was seen in controls with omission of the primary antibodies.

In situ zymography. Following KA treatment, rats were decapitated and their brains were removed, flash frozen, and cryosectioned (12 $\mu \mathrm{m})$. To assess MMP-7 activity, sections were coated with an in situ zymogram assay buffer (50 mM HEPES, $200 \mathrm{~mm} \mathrm{NaCl}, 1 \mathrm{~mm}$ $\mathrm{CaCl}_{2}, 0.01 \%$ Brij-35, pH 7.5) containing $1 \%$ low-melting point agarose and $10 \mu \mathrm{g} / \mathrm{ml}$ MMP-7 fluorogenic substrate (Calbiochem), and then incubated overnight in a humidified chamber at $37^{\circ} \mathrm{C}$. MMP-7 activity was observed using fluorescence microscopy (Nikon) as bright, fluorescent areas on a dark background and calculated as a percentage of the entire hippocampal surface area. Substrate specificity was tested against purified MMP-2, MMP-3, MMP-7, and MMP-9, and activity was only detected in the presence of MMP-7. As a negative control, zymograms from each animal were incubated in the presence of EDTA, a metal chelator that inhibits MMP catalytic activity. No activity was detected in the presence of EDTA.

Fluoro-Jade B labeling. Dying neurons in the hippocampus after KAinduced seizures were assessed by labeling with Fluoro-Jade B (FJ) according to published protocols (Schmued and Hopkins, 2000; Troy et al., 2002).

Western blot analysis. Slices were lysed in RIPA buffer (50 mm Tris/ $\mathrm{HCl}$, pH 7.5, 150 mm NaCl, 5 mm EDTA, 1\% Nonidet P-40, 0.5\% deoxycholic acid, and $0.5 \%$ SDS) supplemented with a protease inhibitor 
mixture (Roche Diagnostics). Total protein was quantified by the Bradford assay (BioRad). Equal amounts of protein from lysates and culture medium were subjected to Western blot analysis and probed with antibodies to MMP-7, TIMP-1, p75, cleaved caspase 3, or NGF. Media samples containing TrkA/Fc were immunoprecipitated using protein A magnetic beads (New England Biolabs). To ensure equal protein loading, blots were stained with Ponceau and stripped/reprobed with actin. All Western blot analyses were performed at least three times with samples from independent experiments.

Statistical analysis. Statistical analysis (Student's $t$ test or ANOVA with Newman-Keuls post hoc analysis) was performed with SigmaStat software (Systat Software). A value of $p<$ 0.05 was taken as statistically significant.

\section{Results}

Kainic acid treatment induces p75 ${ }^{\text {NTR }}$-mediated cell death in organotypic hippocampal slice cultures To examine the regulation of secreted extracellular proneurotrophins in mediating death of hippocampal neurons following kainic acid-induced injury, an organotypic slice culture preparation was initially used. Cultured hippocampal slices were pretreated with MK-801 to block NMDA-mediated toxicity, and then treated for $1 \mathrm{~h}$ with vehicle or $5 \mu \mathrm{M}$ kainic acid, a glutamate agonist that can be used both in vivo and in vitro to evoke injury. Slices were monitored $24 \mathrm{~h}$ later for cell death by measuring PI uptake. KA treatment induced cell death, even in the presence of MK-801, especially throughout the CA1, dentate gyrus, and hilus, as demonstrated by PI uptake that was absent in vehicle-treated slices (Fig. 1a). Kainic acid treatment also induced p $75^{\text {NTR }}$ expression in the same areas, which colocalized with PI labeling (Fig. 1b).

To confirm the role of $\mathrm{p} 75^{\mathrm{NTR}}$ in KAinduced cell death in the hippocampal slices, cultured tissue was lysed and analyzed by Western blot for $\mathrm{p} 75^{\mathrm{NTR}}$ and cleaved caspase 3, a downstream effector of $\mathrm{p} 75^{\mathrm{NTR}}$-mediated apoptosis (Troy et al., 2002). p75 NTR expression levels as well as caspase 3 activation were increased twofold by $8 \mathrm{~h}$ following KA treatment, and this level was sustained through $24 \mathrm{~h}$ (Fig. 1c). To ascertain whether the increased cell death was due to $\mathrm{p} 75^{\mathrm{NTR}}$-mediated signaling, a blocking antibody to $\mathrm{p} 75^{\mathrm{NTR}}$ was applied to hippocampal slices before KA treatment. The $\mathrm{p} 75^{\mathrm{NTR}}$ antibody prevented the increase in cc3 by KA (Fig. 1d), suggesting that, in the absence of NMDA-mediated toxicity, kainic acid-induced death in hippocampal slices is $\mathrm{p} 75^{\mathrm{NTR}}$ dependent.

proNGF levels increase after kainic acid treatment and cause cell death via $\mathrm{p} 75^{\mathrm{NTR}}$

Numerous reports have observed seizure-induced upregulation of NGF mRNA in the hippocampus (Gall and Isackson, 1989; Rocamora et al., 1992; Morimoto et al., 1998; Katoh-Semba et al., 1999). However, these expression studies did not indicate which form of the protein was secreted, or whether proNGF directly functioned as a death-inducing ligand for $\mathrm{p} 75^{\mathrm{NTR}}$ after the sei- zure. To determine which form of NGF was induced and released into the media, hippocampal slices were fixed after treatment and immunostained with an antibody to the pro- region of proNGF (Beattie et al., 2002). ProNGF was induced by kainic acid treatment, particularly in the CA1 and dentate, areas that also demonstrated high levels of PI-positive cells (Fig. 2a). Tissue lysates and medium from kainic acid-treated slice cultures were analyzed by Western blot probed with an antibody to NGF to detect both the mature and pro- forms. ProNGF was detected in both the slice tissue and media, reaching a 2.5 -fold increase in the media by $24 \mathrm{~h}$, indicating that proNGF is produced and secreted from hippocampal cells following kainic acid treatment (Fig. $2 b, c)$. TrkA/Fc chimera was added to the media following KA treatment to sequester any secreted mature NGF for analysis. Although mature NGF was the major form detected in the slice tissue, no mature NGF was detected in the media, even after precipitation with the TrkA/Fc, suggesting that proNGF is the preferentially secreted form of the protein following KA treatment.

To ascertain whether the increased cell death observed in KAtreated slice cultures was due to proNGF activity, a proNGF 
a

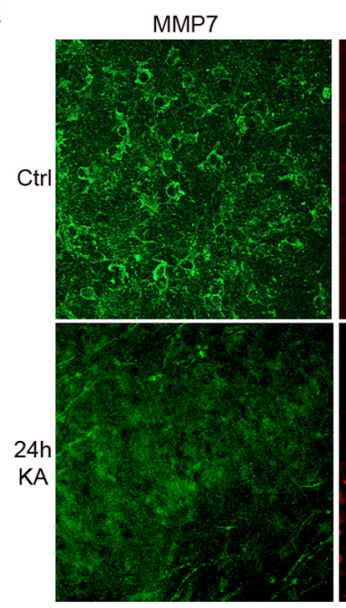

b

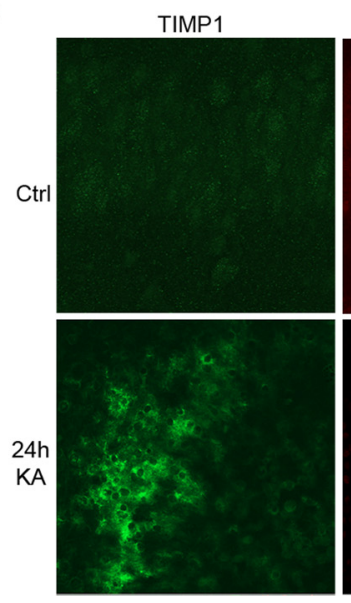

$\mathrm{PI}$
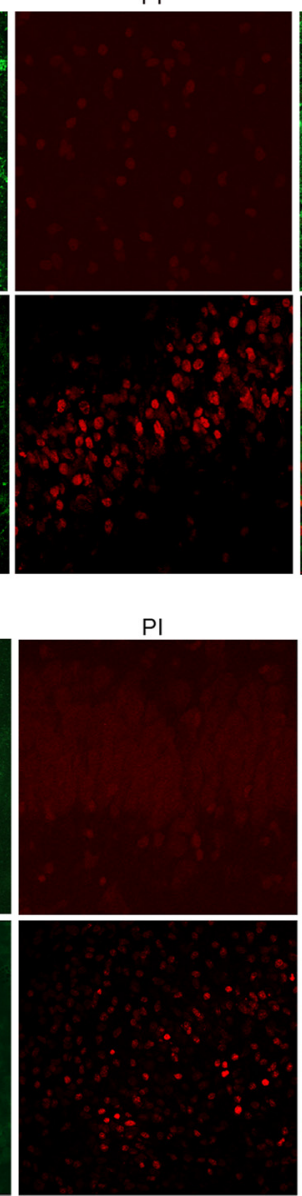

merge
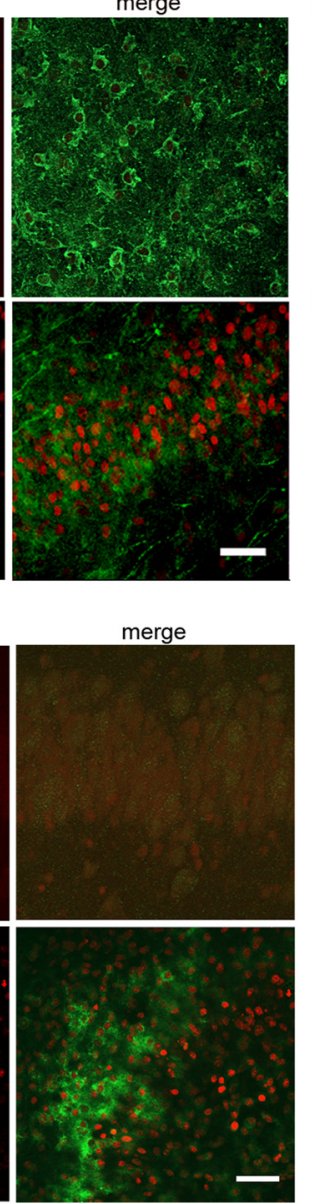

C
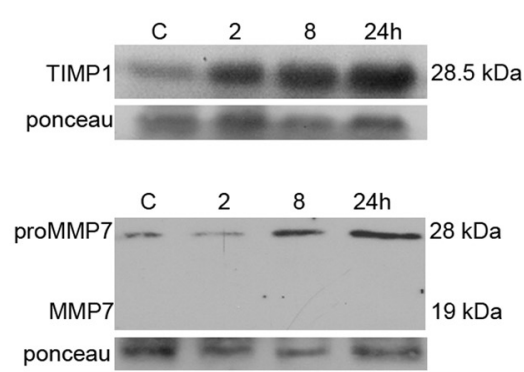

d

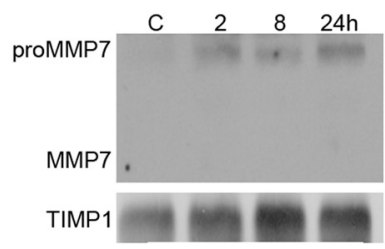

IP: TIMP1

IB: MMP7, TIMP1

Figure 4. Expression of MMP-7 and TIMP-1 in the CA1 following KA treatment in vitro. Following $7 \mathrm{~d}$ in culture, slices were placed into fresh SFM containing $5 \mu \mathrm{g} / \mathrm{ml} \mathrm{PI.} \mathrm{After} 24 \mathrm{~h}$, slices were treated with $5 \mu \mathrm{M} \mathrm{KA}$ for $1 \mathrm{~h}$, and then placed into fresh SFM. Twenty-four hours following treatment, slices were fixed in 4\% PFA, rinsed in PBS, and subjected to immunolabeling with anti-MMP-7 or anti-TIMP-1. $\boldsymbol{a}$, MMP-7 expression is found throughout cell bodies and processes in the hippocampus under basal conditions, but is barely detectable in tissue $24 \mathrm{~h}$ after KA treatment. $\boldsymbol{b}$, TIMP-1 expression is undetected under basal conditions, but is strongly upregulated throughout hippocampal tissue in response to KA treatment. Photographs are from CA1 and are representative of three independent experiments. Scale bar, $50 \mu \mathrm{m}$. c, MMP-7 and TIMP-1 secretion following KA treatment. Western blot analysis of culture medium shows increased secretion of TIMP-1 and proMMP-7 2-24 h following KA treatment. Note mature MMP-7 was undetected. Blots were stained with Ponceau to ensure equal protein loading and transfer. $\boldsymbol{d}$, Medium from slice cultures was immunoprecipitated for TIMP-1 and immunoblotted for MMP-7, and then reprobed for TIMP-1. Extracellular proMMP-7 was detected bound to TIMP-1. Images are representative of four independent experiments.

blocking antibody was applied to culture media before kainic acid treatment. Slices treated with anti-proNGF together with KA demonstrated a $60 \%$ decrease in PI uptake, compared with cultures treated with KA alone (Fig. 3a,b). Western blot analysis also demonstrated that anti-proNGF treatment led a reduction of caspase 3 activation to levels comparable with control (Fig. $3 c, d$ ). These results suggest that proNGF is induced and secreted in response to KA treatment and acts as a death-inducing ligand for p $75^{\text {NTR }}$ in hippocampal slice culture.

\section{MMP-7 and TIMP-1 expression and activity following kainic} acid treatment in vitro

Having demonstrated that proNGF is released from the slice cultures and elicits neuronal death, we investigated the mechanisms that stabilize extracellular proNGF and prevent its cleavage. The expression and release of MMP-7, an enzyme known to cleave proNGF, as well as its inhibitor TIMP-1, was investigated in the hippocampal slice cultures.

To assess whether MMP-7 and TIMP-1 expression patterns were affected in a manner that could facilitate increased proNGF and cell death following KA treatment in slice culture, PI-labeled
KA-treated slices were fixed and immunostained with antibodies to MMP-7 or TIMP-1. MMP-7 expression was observed throughout the hippocampus in normal conditions, but demonstrated a general decrease $24 \mathrm{~h}$ following KA treatment (Fig. $4 a$ ). Conversely, KA induced elevated TIMP-1 expression throughout the CA1 (Fig. 4b).

Western blot analysis of culture media was also performed to measure the amount of protein released into the extracellular environment following KA treatment. Increasing levels of extracellular TIMP-1 and proMMP-7 were both detected following KA treatment, while no mature MMP-7 was observed (Fig. 4c). Since TIMP-1 inhibits MMP-7 by binding to proMMP-7 and preventing its activation by cleavage, we investigated whether secreted TIMP-1 was bound to proMMP-7 in the culture media. Coimmunoprecipiation analysis revealed that the secreted proMMP-7 was bound to TIMP-1. No unbound MMP-7 was detected in the media, suggesting that, although proMMP-7 was released, it was inhibited by TIMP-1 binding (Fig. 4d). These data indicate that following kainic acid-induced injury, proMMP-7 is released from cells, but is bound to TIMP-1, reducing the enzyme activity available for proneurotrophin processing. 
If the endogenous inhibition of MMP-7 contributes to increased proNGF and cell death, then enhancing MMP-7 activity may overcome the endogenous inhibition and promote proNGF cleavage, providing neuroprotection. To determine whether exogenously supplied MMP-7 could prevent neuronal loss, hippocampal slice culture medium was supplemented with $2.5 \mu \mathrm{g}$ of MMP-7 or the equivalent volume of saline $(5 \mu \mathrm{l}) 30 \mathrm{~min}$ before kainic acid treatment. After $24 \mathrm{~h}$, Western blot analysis of culture medium showed that the MMP-7 treatment reduced proNGF to control levels (Fig. $5 a$ ). Furthermore, KA-treated slices in MMP7-supplemented medium demonstrated PI uptake comparable with nontreated controls, indicating that exogenous MMP7 -increased proNGF cleavage can protect cells from KA-induced death in slice culture (Fig. 5b).

\section{MMP-7 activity in the hippocampus is} reduced following seizure

We have previously shown that pilocarpineinduced seizures induce expression of p $75^{\text {NTR }}$ with consequent apoptosis of hippocampal neurons that could be prevented either by using $\mathrm{p} 75^{\mathrm{NTR}}$ knock-out mice (Troy et al., 2002) or by infusing a blocking antibody to proNGF (Volosin et al., 2008), demonstrating that this ligand-receptor system plays a key role in neuronal apoptosis in vivo following seizures. Similar to the effects of seizures induced by pilocarpine, seizures induced by kainic acid also elicited increased $\mathrm{p} 75^{\mathrm{NTR}}$ expression that colocalized with cleaved caspase 3 in hippocampal neurons (Fig. 6).

Having demonstrated that cleavage of extracellular proNGF was regulated by the balance of MMP-7 and TIMP-1 in the medium of slice cultures, we investigated whether MMP-7 expression and activity was also regulated in vivo after KA-induced seizures. Postseizure rats were examined for changes in TIMP-1 and MMP-7 expression to determine whether these protein levels were affected in a manner that could lead to stabilization of extracellular proNGF in vivo. Similar to what we observed in slice cultures, MMP-7 was downregulated in the hippocampus $24 \mathrm{~h}$ after seizure, particularly in the CA1 (Fig. 7a). To assess whether MMP-7 activity also decreased following seizure, in situ zymography was performed on fresh frozen hippocampal sections taken from adult rats with or without kainic acid treatment. Twenty-four hours following seizure, MMP-7 activity displayed a 50\% reduction throughout the hippocampus, particularly in the CA1 and dentate, areas that coincide with seizure-induced cell death (Fig. 7b).

Consistent with decreased MMP-7 activity, TIMP-1 levels were elevated in the hippocampus $24 \mathrm{~h}$ following seizure. Interestingly, TIMP-1 and proNGF were found to be tightly colocal- ized in neurons throughout the CA1 following seizure (Fig. $7 c$, arrows), suggesting that inhibition of MMP-7 may lead to increased proNGF and consequent neuronal death.

\section{MMP-7 protects hippocampal neurons from death following seizure in vivo}

To ascertain whether MMP-7 can reduce the levels of seizureinduced extracellular proNGF in vivo, CSF samples were taken from rats receiving direct infusions of MMP-7 into the hippocampus immediately following KA treatment. The CSF was subjected to Western blot analysis and probed for NGF. ProNGF 

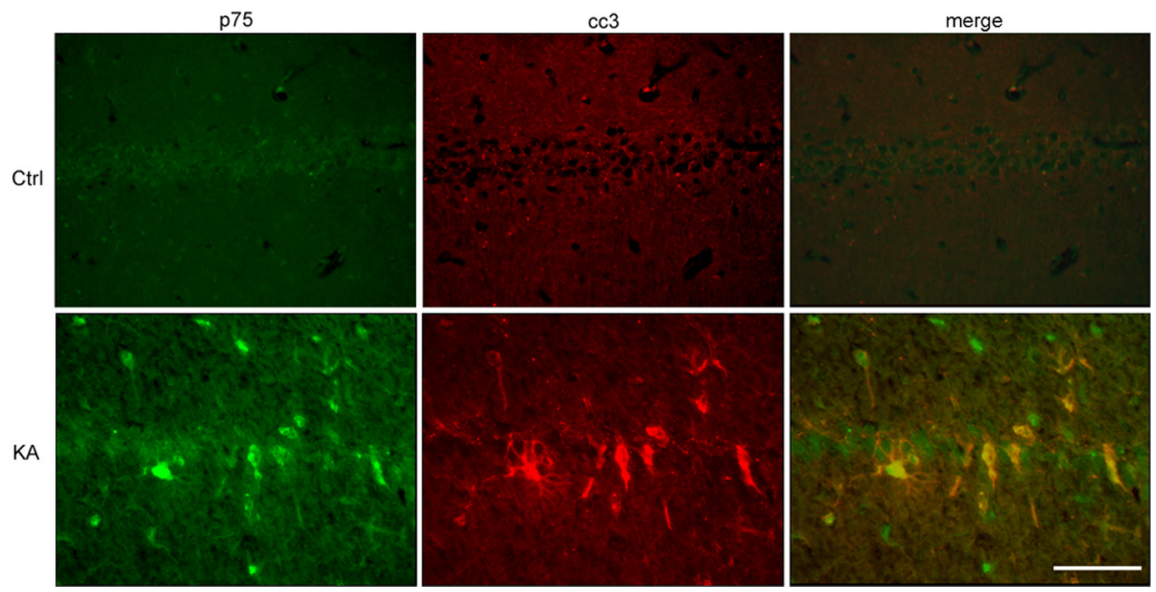

Figure 6. In vivo expression of $75^{\mathrm{NTR}}$ and cleaved caspase 3 increase following kainic acid-induced seizures. Twenty-four hours after induction of SE, rats were anesthetized and brains were prepared for immunocytochemistry. Hippocampal sections were coimmunolabeled with anti-p $75^{\text {NTR }}$ (green) and anti-cc3 (red). The arrows indicate colocalization of p $75^{\text {NTR }}$ and cleaved caspase 3 in response to $K A$-induced seizure.

was not detected in the CSF of rats that were not subjected to seizures, as we have seen previously (Volosin et al., 2008), but was strongly induced in the CSF of rats that underwent KA-induced seizures (Fig. 8a,b). Secreted proNGF levels in KA-treated rats were decreased by $>50 \%$ when MMP-7 was infused into the hippocampus following seizure, suggesting that exogenous MMP-7 leads to the extracellular proteolysis of proNGF.

To determine whether exogenously provided MMP-7 can provide neuroprotection, bilaterally cannulated rats were given a direct infusion of MMP-7 into one hippocampal hemisphere and saline into the other. After $24 \mathrm{~h}$, the rats were killed and their brains were sectioned for cell death analysis using FJ labeling and cleaved caspase 3 immunostaining. In the MMP-7-infused hippocampal hemisphere, FJ labeling was nearly absent compared with the vehicle-infused hemisphere, demonstrating an almost complete rescue from cell death in response to MMP-7 (Fig. 8c). Cells throughout the CA1 of the saline-treated hemisphere demonstrated p $75^{\text {NTR }}$ staining and caspase 3 activation, while detection of $\mathrm{p} 75^{\mathrm{NTR}}$ and cleaved caspase 3 was nearly abolished in the MMP-7-injected hemisphere (Fig. $8 d$ ). Similar results were also observed in the dentate (data not shown). These data suggest that seizures alter the balance of a key cleaving enzyme, MMP-7, and its inhibitor TIMP-1, resulting in reduced MMP-7 activity and increased extracellular proNGF, creating a cellular environment more vulnerable to apoptosis. Restoring MMP-7 reduced the levels of extracellular proNGF and provided neuroprotection after the seizures.

\section{Discussion}

Neurotrophins have long been recognized for their crucial roles in growth, differentiation, and survival in the developing nervous system. Recent findings demonstrating that the pro- form of NGF induces cell death via the $\mathrm{p} 75^{\mathrm{NTR}}$ suggest that the consequences of neurotrophin signaling depend on many factors, in particular the balance between pro- and mature neurotrophins and the regulation of that balance, which may be altered in pathophysiological situations. Since $\mathrm{p} 75^{\mathrm{NTR}}$ is upregulated in many types of neural injury and disease (Park et al., 2000; Troy et al., 2002; Harrington et al., 2004), the increased availability of a ligand that can stimulate apoptotic signaling through this receptor creates an environment in which these neurons are vulnerable. Thus, understanding neurotrophin processing is an important step to- ward understanding the pathway that a cell takes toward survival or death, especially in disease or after injury. We have detected increased proNGF levels in the CSF following seizures, and our data suggest that the increased proNGF promotes neuronal apoptosis. Therefore, it is important to understand the mechanisms that promote stability of proNGF and prevent its cleavage.

We investigated factors in the extracellular environment that can regulate cleavage of the apoptosis-inducing ligand proNGF. Increased levels of proNGF have been detected in the CSF after different types of injury and cause neuronal death under these conditions (Harrington et al., 2004; Volosin et al., 2006). Once released from a cell, proNGF can be processed by MMP-7 (Smith et al., 1995; Lee et al., 2001). However, after injury, we found that proNGF was not only upregulated in the extracellular environment, but its processing enzyme, MMP-7, was decreased in both expression and activity. Moreover, expression of TIMP-1, which inhibits MMP-7, was increased and colocalized with proNGF and with dying neurons following kainic acid treatment, indicative of a functional association between TIMP-1, proNGF, and cell death after injury. In the slice cultures, release of TIMP-1 and proNGF into the media were both dramatically increased following kainic acid treatment, suggesting that proNGF and TIMP-1 are not only upregulated in cells but also released from them as a response to injury. Furthermore, restoring enzyme activity by supplying exogenous MMP-7 in culture or direct infusion into the hippocampus, resulted in decreased extracellular proNGF in the slice media and in the CSF, as well as an almost complete rescue of hippocampal neurons from cell death. These findings suggest that changes to the enzyme-inhibitor ratio may directly affect the balance between pro- and mature NGF after injury, contributing to increased cell death. More specifically, an increase of TIMP-1 after seizure inhibits MMP-7 activity, leading to reduced proteolytic processing of proNGF and thus more available ligand for the p75-mediated apoptotic pathway.

ProNGF can also be cleaved intracellularly by furin and other proconvertases, which would result in the secretion of mature NGF (Seidah et al., 1996). The mechanisms that prevent intracellular proNGF cleavage under specific conditions are unknown. However, it is clear that after injury, elevated levels of extracellular proNGF are detected. MMP-7 is not the only enzyme involved in the extracellular proteolysis of proNGF. The serine protease tissue plasminogen activator (tPA) catalyzes the conversion of the zymogen plasminogen to plasmin, which can also cleave proNGF (Lee et al., 2001; Bruno and Cuello, 2006). Plasminogen, tPA, and its inhibitor, neuroserpin, are released from neurons in conjunction with proNGF upon stimulation, indicating a relationship between the activation of plasmin, the subsequent cleavage of proNGF, and a closely associated inhibitory loop regulated by neuroserpin (Bruno and Cuello, 2006). The tPA-plasmin cascade has also been implicated in neuronal plasticity (Seeds et al., 1995), and in fact, plasmin cleavage of proBDNF to BDNF is essential for hippocampal long-term potentiation (Pang et al., 2004; Nagappan et al., 2009). Although the effects of proBDNF have mostly been examined with regard to physiology, and effects of proNGF have primarily been associated with pathology, this common processing pathway suggests that proper proneurotro- 
phin cleavage plays important roles in several areas of cellular function.

Because proNGF and mature NGF have differential effects on neuronal survival, and because proNGF levels increase in parallel with neuronal apoptosis following various forms of injury or pathological conditions, the processing of proto mature NGF is critical in determining neuronal fate. The enzymatic cleavage of proNGF to NGF by MMP-7 appears to be important for neuronal survival following seizures and possibly other types of injury in which proNGF is elevated (Beattie et al., 2002; Pang et al., 2004; Volosin et al., 2006). However, MMP-7 and TIMP-1 activity in the brain remain relatively unexplored. Changes in MMP-7 expression have been detected in an animal model of multiple sclerosis (Buhler et al., 2009) and in the brains of Alzheimer's patients (Ethell et al., 2002). If alterations to the MMP-7/TIMP-1 balance contribute to the increased proNGF and improper trophic support seen in Alzheimer's and other neurodegenerative disorders, it will be crucial to continue investigating the influence of enzymatic regulation and the potential outcomes of altering enzyme activity on neurotrophin cleavage, and the consequences for neuronal survival or death. Our findings on the relationship between MMP-7 and proNGF following seizure-induced injury demonstrate that the regulation of enzymatic processing of proNGF by MMP-7 is important for cell survival and suggest a novel mechanism by which MMP-7 may function in the injury process.

\section{References}

Ali TK, Al-Gayyar MM, Matragoon S, Pillai BA, Abdelsaid MA, Nussbaum JJ, El-Remessy AB (2011) Diabetes-induced peroxynitrite impairs the balance of pro-nerve growth factor and nerve growth factor, and causes neurovascular injury. Diabetologia 54:657-668.

Beattie MS, Harrington AW, Lee R, Kim JY, Boyce SL, Longo FM, Bresnahan JC, Hempstead BL, Yoon SO (2002) ProNGF induces p75-mediated death of oligodendrocytes following spinal cord injury. Neuron 36:375-386.

Birkedal-Hansen H (1993) Role of matrix metalloproteinases in human periodontal diseases. J Periodontol 64:474-484.

Bruno MA, Cuello AC (2006) Activity-dependent release of precursor nerve growth factor, conversion to mature nerve growth factor, and its degradation by a protease cascade. Proc Natl Acad Sci U S A 103:6735-6740.

Buhler LA, Samara R, Guzman E, Wilson CL, Krizanac-Bengez L, Janigro D, Ethell DW (2009) Matrix metalloproteinase-7 facilitates immune access to the CNS in experimental autoimmune encephalomyelitis. BMC Neurosci 10:17. a
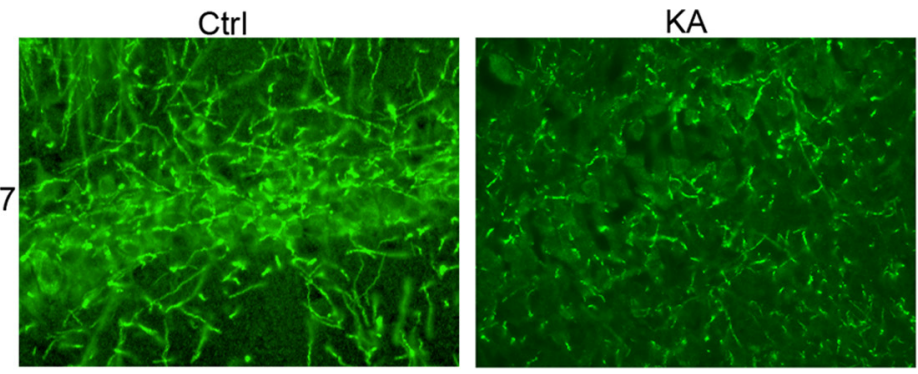

b


C



Figure 7. Expression of MMP-7, TIMP-1, and proNGF $24 \mathrm{~h}$ following KA-induced seizure in vivo. a, Hippocampal sections from postseizure rats immunolabeled with anti-MMP-7. Vehicle-treated rats demonstrated high levels of MMP-7 expression throughout cell bodies and processes, but this was greatly diminished throughout the hippocampus after seizure. $\boldsymbol{b}$, MMP-7 activity was reduced in response to KA-induced seizure. Fresh frozen sections were coated with an in situ zymogram assay buffer containing $10 \mu \mathrm{g} / \mathrm{ml} \mathrm{MMP-7} \mathrm{fluorogenic} \mathrm{substrate} \mathrm{that} \mathrm{is} \mathrm{specific} \mathrm{to} \mathrm{MMP-7} \mathrm{activity.} \mathrm{MMP-7} \mathrm{activity} \mathrm{demon-}$ strated a $60 \%$ reduction in the hippocampus, particularly in the CA1 and dentate, areas that coincide with seizure-induced cell death. Activity was calculated as relative fluorescence intensity of the entire hippocampal surface area, normalized to background fluorescence. $N=5$ independent experiments, and error bar represents SEM. Data were analyzed using Student's $t$ test. ${ }^{* *} p<0.001$. In situ zymogram performed in the presence of EDTA, a metal chelator that inhibits MMPs, demonstrated abolished activity. Scale bar, $100 \mu \mathrm{m}$. c, ProNGF (green) and TIMP-1 (red) were strongly induced and were found in some of the same cells throughout the CA1 following KA-induced seizure. Images are representative of five independent experiments. Scale bars, $50 \mu \mathrm{m}$. 
a

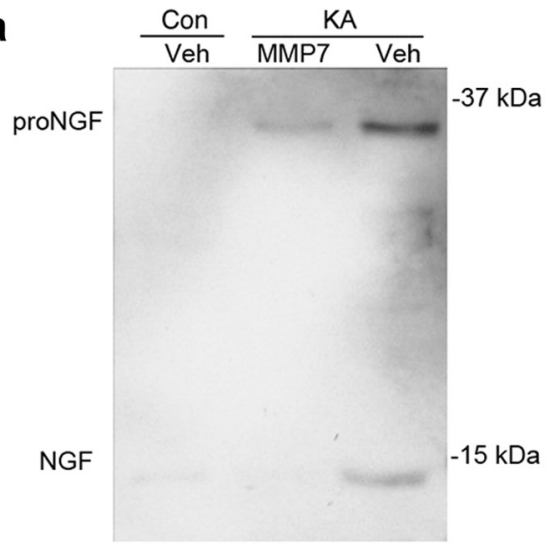

C

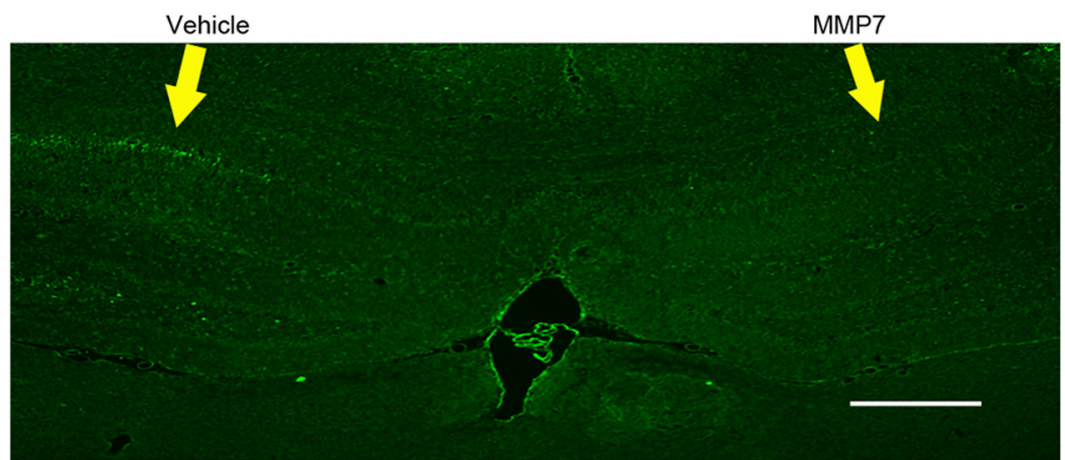

d
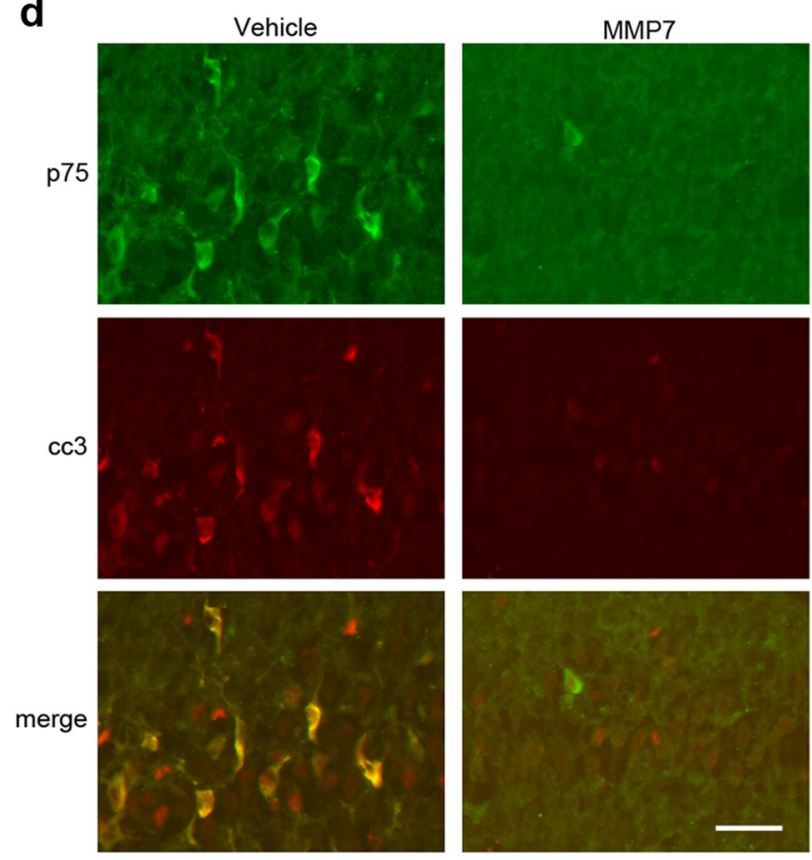

Figure 8. Exogenous MMP-7 reduces extracellular proNGF and protects hippocampal neurons from death following seizure in vivo. $\boldsymbol{a}$, Ten micrograms of CSF protein from cannulated rats with or without KA treatment were analyzed by Western blot. Rats that received KA with vehicle infusion showed a threefold increase in extracellular proNGF compared with control rats. MMP-7 infusion induced a 54\% reduction in extracellular proNGF compared with those that received vehicle infusions. $\boldsymbol{b}$, Graph represents the normalized densitometric index of blots from three independent experiments. Error bars represent SEM. Data were analyzed using one-way ANOVA, followed by Student-Newman-Keuls post hoc test. ${ }^{* *} p=0.002$, ${ }^{* *} p<0.001$. c, Low-magnification image from a bilaterally cannulated, seizure-induced rat that received infusions of MMP-7 $(1 \mu \mathrm{g})$ into one hippocampal hemisphere, and vehicle $(0.5 \mu \mathrm{l})$ into the opposite hemisphere, immediately following seizure treatment and twice a day thereafter. Decreased Fluoro-Jade B staining was observed in the hippocampal hemisphere ipsilateral to MMP-7 injection (arrows). Scale bar, $100 \mu \mathrm{m}$. $\boldsymbol{d}$, High-magnification sections showing immunostaining for $\mathrm{p} 75^{\mathrm{NTR}}$ and cleaved caspase 3. The left column represents the vehicleinfused side, and the right column represents the MMP-7-infused side. Twenty-four hours following seizure, immunostaining for cleaved caspase 3 and $p 75^{\text {NTR }}$ was diminished on the MMP-7-infused side compared with the vehicle-infused hemisphere, indicating
Casaccia-Bonnefil P, Carter BD, Dobrowsky RT, Chao MV (1996) Death of oligodendrocytes mediated by the interaction of nerve growth factor with its receptor p75. Nature 383:716-719.

Ethell DW, Kinloch R, Green DR (2002) Metalloproteinase shedding of Fas ligand regulates beta-amyloid neurotoxicity. Curr Biol $12: 1595-1600$

Friedman WJ (2000) Neurotrophins induce death of hippocampal neurons via the p75 receptor. J Neurosci 20:6340-6346.

Gall CM, Isackson PJ (1989) Limbic seizures increase neuronal production of messenger RNA for nerve growth factor. Science 245:758-761.

Giehl KM, Röhrig S, Bonatz H, Gutjahr M, Leiner B, Bartke I, Yan Q, Reichardt LF, Backus C, Welcher AA, Dethleffsen K, Mestres P, Meyer M (2001) Endogenous brain-derived neurotrophic factor and neurotrophin-3 antagonistically regulate survival of axotomized corticospinal neurons in vivo. J Neurosci 21:3492-3502.

Gogly B, Fournier B, Couty L, Naveau A, Brasselet C, Durand E, Coulomb B, Lafont A (2009) Gingival fibroblast inhibits MMP-7: evaluation in an ex vivo aorta model. J Mol Cell Cardiol 47:296-303.

Harrington AW, Leiner B, Blechschmitt C, Arevalo JC, Lee R, Mörl K, Meyer M, Hempstead BL, Yoon SO, Giehl KM (2004) Secreted proNGF is a pathophysiological death-inducing ligand after adult CNS injury. Proc Natl Acad Sci U S A 101:6226-6230.

Jourquin J, Tremblay E, Bernard A, Charton G, Chaillan FA, Marchetti E, Roman FS, Soloway PD, Dive V, Yiotakis A, Khrestchatisky M, RiveraS (2005) Tissue inhibitor of metalloproteinases-1 (TIMP-1) modulates neuronal death, axonal plasticity, and learning and memory. Eur J Neurosci 22:2569-2578.

Katoh-Semba R, Takeuchi IK, Inaguma Y, Ito H, Kato K (1999) Brain-derived neurotrophic factor, nerve growth and neurotrophin-3 selected regions of the rat brain following kainic acid-induced seizure activity. Neurosci Res 35:19-29.

Kendall TJ, Hennedige S, Aucott RL, Hartland $\mathrm{SN}$, Vernon MA, Benyon RC, Iredale JP (2009) p75 Neurotrophin receptor signaling regulates hepatic myofibroblast proliferation and apoptosis in recovery from rodent liver fibrosis. Hepatology 49:901-910.

Lee R, Kermani P, Teng KK, Hempstead BL (2001) Regulation of cell survival by secreted proneurotrophins. Science 294:1945-1948.

Levi-Montalcini R (1964) Growth control of nerve cells by a protein factor and its antiserum: discovery of this factor may provide new leads to understanding of some neurogenetic processes. Science 143:105-110.

Levi-Montalcini R (1987) The nerve growth factor 35 years later. Science 237:1154-1162.

Morimoto K, Sato K, Sato S, Yamada N, Hayabara $\mathrm{T}$ (1998) Time-dependent changes in neurotrophic factor mRNA expression after kin-

MMP-7 protects against KA-induced, p75 ${ }^{\text {NTR }}$-mediated cell death. Images are from the CA1 area of the hippocampus and are representative of five independent experiments. Scale bar, $50 \mu \mathrm{m}$. 
dling and long-term potentiation in rats. Brain Res Bull 45:599-605.

Nagappan G, Zaitsev E, Senatorov VV Jr, Yang J, Hempstead BL, Lu B (2009) Control of extracellular cleavage of ProBDNF by high frequency neuronal activity. Proc Natl Acad Sci U S A 106:1267-1272.

Pang PT, Teng HK, Zaitsev E, Woo NT, Sakata K, Zhen S, Teng KK, Yung WH, Hempstead BL, Lu B (2004) Cleavage of proBDNF by tPA/plasmin is essential for long-term hippocampal plasticity. Science 306:487-491.

Park JA, Lee JY, Sato TA, Koh JY (2000) Co-induction of p75NTR and p75NTR-associated death executor in neurons after zinc exposure in cortical culture or transient ischemia in the rat. J Neurosci 20:9096-9103.

Parks WC, Shapiro SD (2001) Matrix metalloproteinases in lung biology. Respir Res 2:10-19.

Paxinos G, Watson C (1986) The rat brain in stereotaxic coordinates, Ed 2. San Diego: Academic.

Peng S, Wuu J, Mufson EJ, Fahnestock M (2004) Increased proNGF levels in subjects with mild cognitive impairment and mild Alzheimer disease. J Neuropathol Exp Neurol 63:641-649.

Racine RJ (1972) Modification of seizure activity by electrical stimulation. II. Motor seizure. Electroencephalogr Clin Neurophysiol 32:281-294.

Rivera S, Tremblay E, Timsit S, Canals O, Ben-Ari Y, Khrestchatisky M (1997) Tissue inhibitor of metalloproteinases-1 (TIMP-1) is differentially induced in neurons and astrocytes after seizures: evidence for developmental, immediate early gene, and lesion response. J Neurosci 17:4223-4235.

Rocamora N, Palacios JM, Mengod G (1992) Limbic seizures induce a differential regulation of the expression of nerve growth factor, brainderived neurotrophic factor and neurotrophin-3, in the rat hippocampus. Brain Res Mol Brain Res 13:27-33.

Schmued LC, Hopkins KJ (2000) Fluoro-Jade B: a high affinity fluorescent marker for the localization of neuronal degeneration. Brain Res 874: $123-130$.
Seeds NW, Williams BL, Bickford PC (1995) Tissue plasminogen activator induction in Purkinje neurons after cerebellar motor learning. Science 270:1992-1994.

Seidah NG, Benjannet S, Pareek S, Savaria D, Hamelin J, Goulet B, Laliberte J, Lazure C, Chrétien M, Murphy RA (1996) Cellular processing of the nerve growth factor precursor by the mammalian pro-protein convertases. Biochem J 314:951-960.

Smith MM, Shi L, Navre M (1995) Rapid identification of highly active and selective substrates for stromelysin and matrilysin using bacteriophage peptide display libraries. J Biol Chem 270:6440-6449.

Stoppini L, Buchs PA, Muller D (1991) A simple method for organotypic cultures of nervous tissue. J Neurosci Methods 37:173-182.

Tan HK, Heywood D, Ralph GS, Bienemann A, Baker AH, Uney JB (2003) Tissue inhibitor of metalloproteinase 1 inhibits excitotoxic cell death in neurons. Mol Cell Neurosci 22:98-106.

Troy CM, Friedman JE, Friedman WJ (2002) Mechanisms of p75-mediated death of hippocampal neurons. Role of caspases. J Biol Chem 277: 34295-34302.

Volosin M, Song W, Almeida RD, Kaplan DR, Hempstead BL, Friedman WJ (2006) Interaction of survival and death signaling in basal forebrain neurons: roles of neurotrophins and proneurotrophins. J Neurosci 26:7756-7766.

Volosin M, Trotter C, Cragnolini A, Kenchappa RS, Light M, Hempstead BL, Carter BD, Friedman WJ (2008) Induction of proneurotrophins and activation of p75NTR-mediated apoptosis via neurotrophin receptorinteracting factor in hippocampal neurons after seizures. J Neurosci 28:9870-9879.

Wojtowicz-Praga SM, Dickson RB, Hawkins MJ (1997) Matrix metalloproteinase inhibitors. Invest New Drugs 15:61-75. 